\title{
Multimode Interference as a Tool for Fiber Sensing
}

\author{
J. L. Santos ${ }^{\mathrm{a}, \mathrm{b}}$, Susana Silva, ${ }^{\mathrm{a}, \mathrm{b}}$, L. A. Ferreira ${ }^{\mathrm{a}}$, F. M. Araújo ${ }^{\mathrm{a}}$, O. Frazão ${ }^{\mathrm{a}}$ \\ ${ }^{a}$ INESC Porto, Rua do Campo Alegre 687, 4169-007 Porto, Portugal \\ ${ }^{b}$ Departamento de Física e Astronomia da Faculdade de Ciências da Universidade do Porto, \\ Rua do Campo Alegre 687, 4169-007 Porto, Portugal
}

\begin{abstract}
Fiber optic modal interferometry has been around as a sensing concept since the outcome of fiber optic sensing. Initially supported by the utilization of standard Hi-Bi fibres associated to polarimetric modal interference, later this sensing approach evolved to modal interference based on spatial modes propagating in the core, on spatial modes propagating in the core and in the cladding with coupling performed by fibre devices such as long period gratings and tapers, and more recently on several types of modes propagating in photonic crystal fibers. This paper will address fiber optic sensing based on modal interferometry, and configurations of different type researched in last years will be presented and their performance compared.
\end{abstract}

Keywords: Fiber Optics, Modal Interference, Sensing.

\section{HISTORIC OVERVIEW}

Modal interferometry in optical fibers deals with the differential phase between two (or more) fiber modes, with the sensing opportunity associated with the possibility this phase to change induced by a specific measurand under controlled conditions. This type of interferometry exhibits a substantial common mode rejection, which means a substantial reduction of environmental susceptibility. Add to this the simplicity and compactness of these structures and, naturally, turns out the interest of the R\&D community in their study and to address the feasibility of their application in diverse situations.

There are two types of fiber modes that can be considered: spatial modes and polarization modes. Within the first type, there is possibility to combine core spatial modes or core-cladding spatial modes. In the former case, interference of LP modes is involved (for example, $\mathrm{LP}_{01}$ and $\mathrm{LP}_{11}$ in elliptical core fibers). Indeed, the first report of optical fiber sensing based on modal interferometry (that goes back to 1979) uses this approach for acoustic detection, specifically interference of $\mathrm{HE}_{11}$ and $\mathrm{TM}_{01}$ modes (Layton et al [1]). However, it requires particular launch and propagation conditions, which is difficult to achieve, with the consequence such possibility along the years has been more of academic interest. When considering core-cladding modes, the essential feature is to have a device that can couple light from the core mode to one of the cladding modes and the other way around. Initially, this was done playing with the splicing between two fibers or with tapers. However, typically the insertion losses were high and it was difficult to do selective coupling between the core mode and a specific cladding mode. With the outcome of fiber gratings, the situation changed drastically and nowadays this is one of the favorite approaches to implement multimode interference for sensing in view of its simplicity and effectiveness, as well as the fact the sensing structure becomes sensitive to the external environment.

Multimode interference for sensing based one polarimetric modes was first demonstrated by Eickhoff in 1981 [2], as a consequence of the recent availability of high birefringent fibers. Sensor structures based on this approach are fairly easy

Fiber Optic Sensors and Applications IX, edited by Henry H. Du, Gary Pickrell, Eric Udd,

Jerry J. Benterou, Alexis Mendez, Stephen J. Mihailov, Anbo Wang, Proc. of SPIE Vol. 8370, 83700B

(C) 2012 SPIE · CCC code: $0277-786 X / 12 / \$ 18 \cdot$ doi: $10.1117 / 12.919974$

Proc. of SPIE Vol. $837083700 B-1$ 
to control, therefore along the years many configurations relying on the interference of polarimetric modes have been researched and applied in a diversity of situations.

It has also been explored modal interference involving more than two modes, resulting into a spectral transfer function that is no more co-sinusoidal but instead show sharp peaks at specific wavelengths. It is common to refer this approach as multimode interference (MMI), and several layouts have been researched. It is the case of the structure studied in 2003 by Mehta et al [3]. The theoretical model and experimental setup of a displacement sensor was demonstrated. The structure consisted in splicing a multimode fiber (MMF) section between two single mode fibers (SMFs). The end of the MMF was cleaved and combined with a planar mirror to form the displacement sensor. In 2006, Jung et al [4] proposed a compact surrounding refractive-index sensor using an MMF-coreless silica fiber-MMF structure where the evanescent waves in the coreless silica fiber region could directly interact with the surrounding medium. The suggested device has shown high potential for a wide range of biomedical and chemical sensor applications. In the same year, Li et al [5] developed a fiber-optic temperature sensor in reflection that relied on the interference between selective higher-order modes in multimode optical fibers. It was demonstrated that coupling the $\mathrm{LP}_{01}$ mode in a standard SMF to the $\mathrm{LP}_{0 \mathrm{~m}}$ modes in an MMF, and utilizing the interference of the higher-order modes, a fiber-optic high-temperature sensor could be obtained. In another perspective, Mohammed et al [6] reported a fixed-wavelength bandpass filter based on multimode interference and reimaging concept. It was investigated various factors such as MMF length and geometries of the SMF and MMF that affected the filter center wavelength, bandwidth, and isolation. It was shown that the bandwidth of the device changed when the strain was applied. Later, Wang et al [7] employed a modeling tool for simulation and design of a similar structure as an optical fiber refractometer sensor. In 2007, Frazão et al [8] demonstrated an all fiber Mach-Zehnder interferometer based on a MMI-based fiber structure combined with a longperiod fiber grating (LPG). In 2009, Zhang et al [9] studied a sensing scheme to measure temperature and strain simultaneously by multiplexing a section of an MMF and a Fizeau etalon interferometer. In 2010, Zhang et al [11] reported a refractometer based on a SMS fiber structure. The HF corrosion method was also applied in order to improve the sensitivity of the sensing device. Recently, a simple displacement sensor based on a bent SMS fiber structure was experimentally investigated by $\mathrm{Wu}$ et al [12]. Moreover, Silva et al [13] characterized a SMS structure based on a largecore, air-clad photonic crystal fiber as a refractometer sensor. Using two distinct large-core air-clad PCF geometries, one for refractive index measurement and other for temperature compensation, it was possible to implement a sensing head that was sensitive to refractive index changes induced by temperature variations in water as base matrix. Also, using the fiber optic sensor configuration developed by Li et al [5], a temperature-independent refractometer sensor could be achieved [13]. In another perspective, a temperature and strain independent curvature sensor was attained by means of the SMS fiber structure technology [14].

An overview of the various types of MMI-based fiber optic structures in a chronological order is presented in the following Table.

Chronology of MMI-Based Optical Fiber Sensors.

\begin{tabular}{llr}
\hline Structure Device & Sensor Type & References \\
SMF-MMF & Displacement & {$[3]$} \\
MMF-Coreless Silica Fiber-MMF & Refractometer & {$[4]$} \\
SMF-MMF-Mirror & High-temperature & {$[5]$} \\
SMF-MMF-SMF & Strain & {$[6]$} \\
SMF-MMF-SMF & Refractometer & {$[7]$} \\
SMF-MMF-LPG & Curvature & {$[8]$} \\
SMF-MMF-Fizeau & Simultaneous measurement of strain and temperature & {$[9]$} \\
SMF-MMF-SMF & Refractometer and corrosion & {$[10]$} \\
SMF-LC-Air Clad PCF-SMF & Displacement and bent & {$[11]$} \\
SMF-MM PCF-SMF & Refractometer & {$[12]$} \\
SMF-MMF & Temperature-independent refractometer & {$[13]$} \\
SMF-MMF-SMF & Temperature and strain independent curvature sensor & {$[14]$} \\
\hline
\end{tabular}


It is important to mention that the availability of Photonic Crystal Fibers brought a breakthrough in the domain of modal interferometry for sensing in any of the modal combinations (polarization modes, core spatial modes and core-cladding spatial modes), essentially in view of design flexibility and low-temperature sensitivity of modal sensing structures supported by these fibers. The consequence was a burst of R\&D activity in fiber sensing.

\section{MULTIMODE INTERFERENCE SENSING CONFIGURATIONS}

In this section three examples are given of fiber optic sensing structures based on modal interferometry. The first one reports a Michelson modal interferometer assisted by a single non-adiabatic taper [15], the second deals with a MachZehnder modal structure combined with a Long Period Grating (LPG) [16], and the third one addresses the effect of fiber tapering in modal LPG-based Mach-Zehnder interferometers [17].

\subsection{Modal Interferometer Based on a Single Non-adiabatic Fibre Taper}

The motivation to work out a taper sensing structure was the perception that would be advantageous to have a modal fibre interferometer with the coupling to the cladding of a fraction of the core light performed by a structure easier to fabricate than a LPG. It is shown here such objective is possible with the sensing head geometry shown in Figure 1 . The non-adiabatic taper was fabricated in Corning SMF-28 fibre using a splice machine combined with fibre elongation during the arc discharge. The fabrication parameters were adjusted in order to decrease the fibre diameter from $125 \mu \mathrm{m}$ down to $80 \mu \mathrm{m}$ in the taper waist. The total length of the taper was and the insertion loss were $\sim 500 \mu \mathrm{m}$ and $\sim 3 \mathrm{~dB}$, respectively.
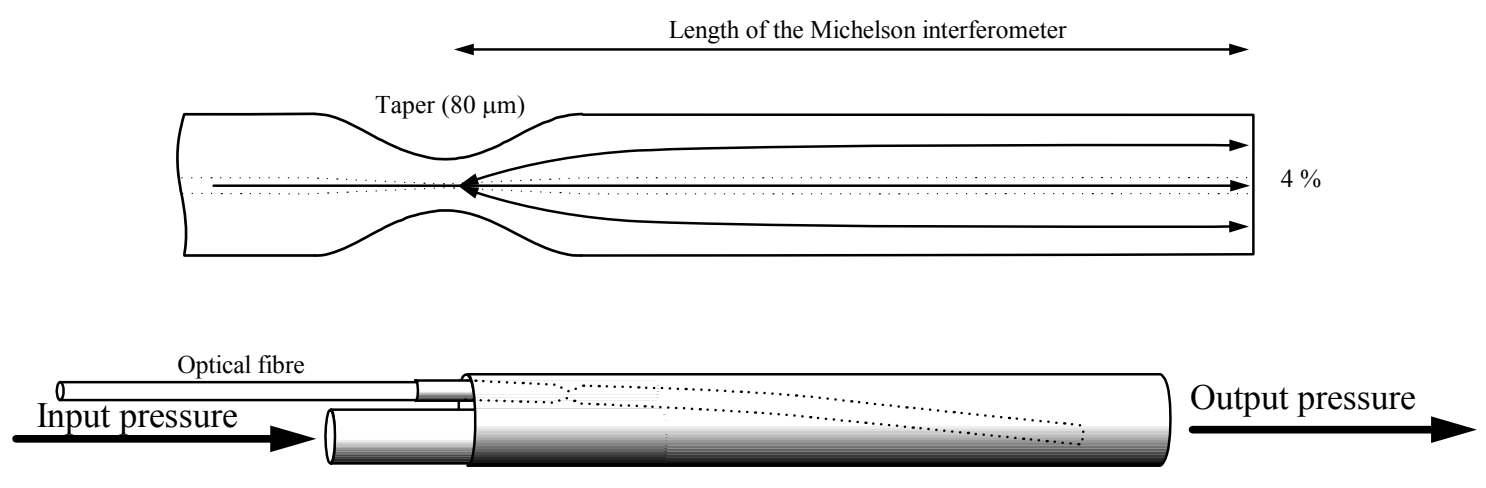

Figure 1. Geometry of the Michelson modal interferometer and the optical flowmeter design.

The fibre length after the taper is $\mathrm{L} \sim 100 \mathrm{~mm}$ and the reflection coefficient at the fibre end is that of an air-glass interface $(\sim 4 \%)$. The effect of the optical taper is to expand the core mode field and to couple light into the cladding modes, which propagates up to the end of the fibre. The fraction of the light that is coupled to the cladding depends on the taper length and depth, and in the present case these parameters were chosen to have a high fringe visibility in the interferometric structure. The fraction of the light that is reflected back goes again through the taper, which induces a recombination effect, originating light waves that propagate down the fibre coming from the core and cladding regions in the sensing length. Due to the difference in the effective refractive indexes, these waves accumulate an optical path difference of $290 \mu \mathrm{m}$, value obtained from the tuning of the receiver interferometer as described below. This value is substantially larger than the coherence length, $L_{c}$, of the optical source, which was a superluminescent diode that emits at $1320 \mathrm{~nm}$ with a spectral width of $\sim 35 \mathrm{~nm}$, resulting in $\mathrm{L}_{\mathrm{c}} \approx 33 \mu \mathrm{m}$. 
As shown in Figure 2, the returned light is injected into a receiver Michelson interferometer with an open air path in one of the arms, which is used to tune the optical path difference of this interferometer to that of the sensing modal interferometer. In this way coherence addressing is performed and an interferometric signal appears at the input of the photodetector [18]. Applying a sawtooth waveform with specific amplitude to a PZT fibre stretcher in one arm of the interferometer, with proper electrical filtering an electric carrier is obtained with a phase that mirrors the phase of the tandem interferometric system. This phase was measured using a lock-in amplifier.

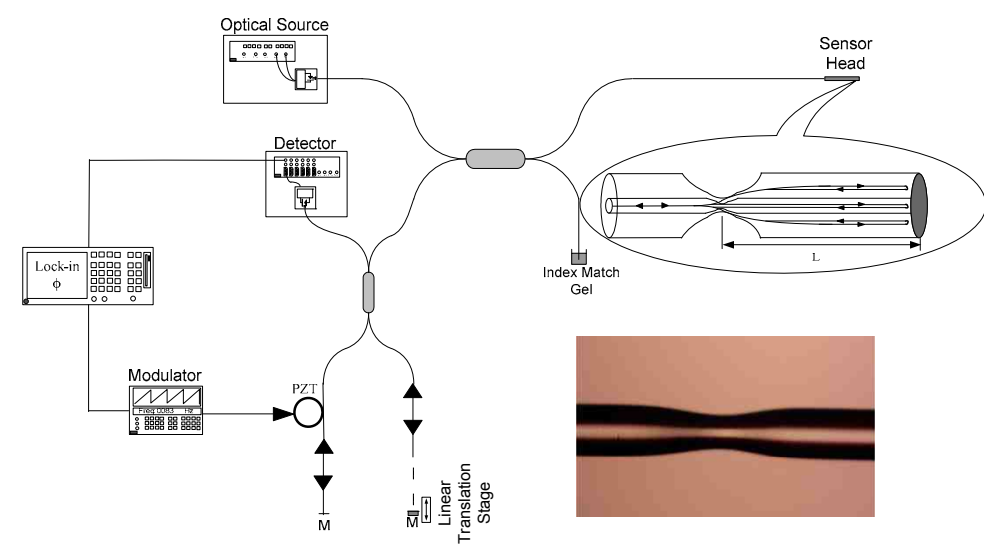

Figure 2. Scheme of the sensing system (inset: photo of the fibre taper).

Figure 3 shows the reflected power spectrum for the taper-assisted interferometer at the input of the receiving interferometer. Some important features can be drawn from this result. First, besides the presence of some fine structure in the spectrum, an indication of some degree of multiple-wave interference, it shows essentially the typical behavior of the channeled spectrum of a two-wave interferometer. Therefore, the recombination at the taper after reflection at the fibre end involves essentially the light from the core mode and from one of the cladding modes. Indeed, from the spectral periodicity of the fringes in Figure $3(\sim 6 \mathrm{~nm})$ and the length of the sensing fibre $(100 \mathrm{~mm})$, it turns out that $n_{\text {core }}-n_{\text {cladding }} \approx 1.45 \times 10^{-3}$, which means that the interference is mostly between the light in the $\mathrm{LP}_{01}$ core mode and the light in the $\mathrm{LP}_{02}$ cladding mode. Also, the visibility of the interferometric fringes reaches $75 \%$, which shows the effectiveness of the short non-adiabatic taper to induce interference. Finally, the level of reflected power could be increased substantially by mirroring the end of the sensing fibre to reach a power reflectivity close to $100 \%$.

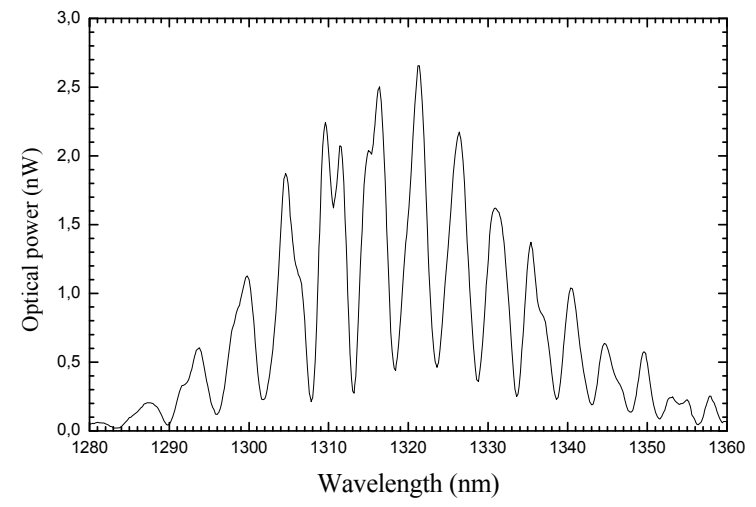

Figure 3. Reflected power spectrum from the taper-assisted modal interferometer. 
It was observed that the interferometric phase is highly sensitive to the inclination of the fibre after the taper. Indeed, the own weight of this fibre induces an inclination from the horizontal position which originates a change in the interferometric phase of the order of $150^{\circ}$. Therefore, this structure can be used as highly sensitive curvature sensor. However, in this work we choose to take advantage of this characteristic to build up a flowmeter. The concept is illustrated in Figure 1. With the geometry shown, without flow of air the sensing fibre is under maximum inclination due to its weight. This inclination is reduced when the flow increases, or equivalently, with the increase of the input pressure. Therefore, the interferometric phase shall have a dependence with this pressure (compressed air in the range 0.5-3 bar), which is confirmed by the data shown in Figure 4, where it is clear a linear relation between the two parameters (slope of 69.8 degrees/bar, with a maximum error of $\sim 3 \%$ ).

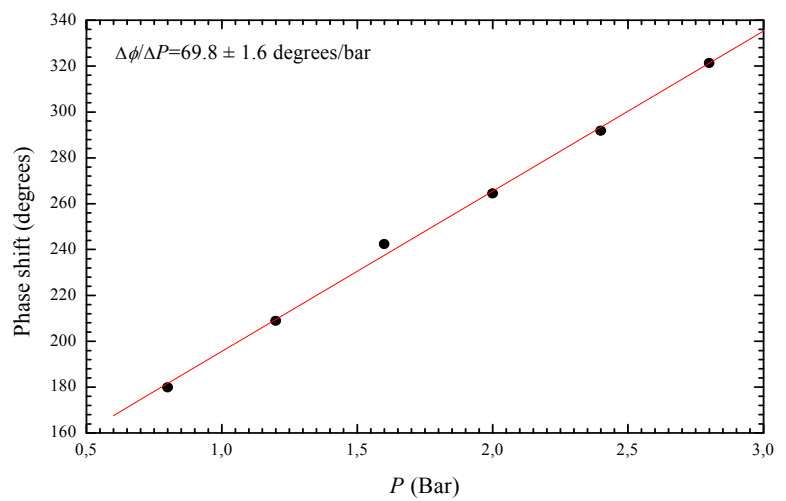

Figure 4. Interferometer phase change versus input pressure in the configuration shown in Figure 1.

To have an optical flowmeter it is necessary to have calibration data between the input pressure and the air flow. This was obtained using a conventional flowmeter which gave: flow velocity in $\mathrm{ml} / \mathrm{min}(\mathrm{v})=-7845.8+12198.3 P-1606.6 P^{2}$, with $P$ in bar (inset of Figure 5). With this information and the data of Figure 4, it is possible to have a relation between optical phase variation and flow velocity, which is given in Figure 5.

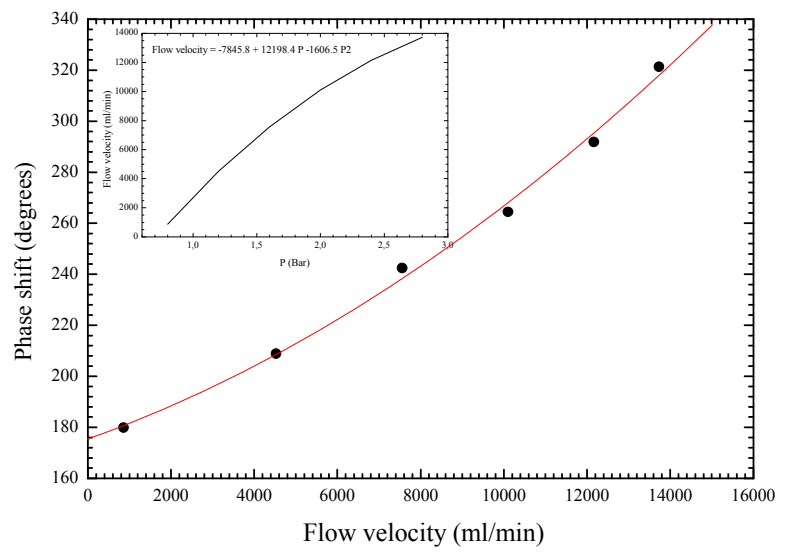

Figure 5. Interferometer phase change versus flow velocity (inset figure: calibration curve between input pressure in Figure 1 and flow velocity).

This type of modal interferometer is very simple to build. In addition, it is substantial its potential for high sensitive measurement of a large range of parameters, as was demonstrated here concerning the determination of flow velocity. 


\subsection{In-Fiber Mach-Zehnder Configuration Based on Multimode Interference Combined with a LPG}

In this section it is presented a Mach-Zehnder interferometer using multimode interference combined with a long period fibre grating (MMI-LPG), with demonstration of its application as a bending sensor.

For tuning the optimum multimode fibre length, a 3D simulation using Beam Propagation Method (BPM) was performed on Rsoft's BPMCAD. Light from a single-mode fibre SMF28 was injected in the multimode fibre with the following characteristics: numerical aperture (N.A.) of 0.22 , core and cladding diameters are $105 \mu \mathrm{m}$ and $125 \mu \mathrm{m}$, respectively. For the operational center wavelength of $1324 \mathrm{~nm}$, the refractive index of the assumed pure $\mathrm{SiO}_{2}$ cladding was interpolated based on data from literature. A single mode fibre (SMF 28) was used as the input and output of the multimode fibre.

The intensity distribution of the electric field is plotted in Figure 6, on the left, for XZ-cut, where $\mathrm{X}$ is a radial coordinate and $\mathrm{Z}$ the axial one. On the right, is plotted the coupling overlap integral between the calculated field at a given multimode fibre length and the input field for the simulation, a SMF-28 fundamental mode. It can be seen that optimal coupling into the output SMF-28 is achieved sharply around $38.7 \mathrm{~mm}$. For our device implementation, a coupling ratio between core and cladding modes of 0.5 is advisable, so the optimal setting is around $32 \mathrm{~mm}$.

The mode profile coupled into the output single-mode fibre is shown in inset Figure 6 . Almost $50 \%$ of the input is coupled into the core of the SMF-28 fibre.
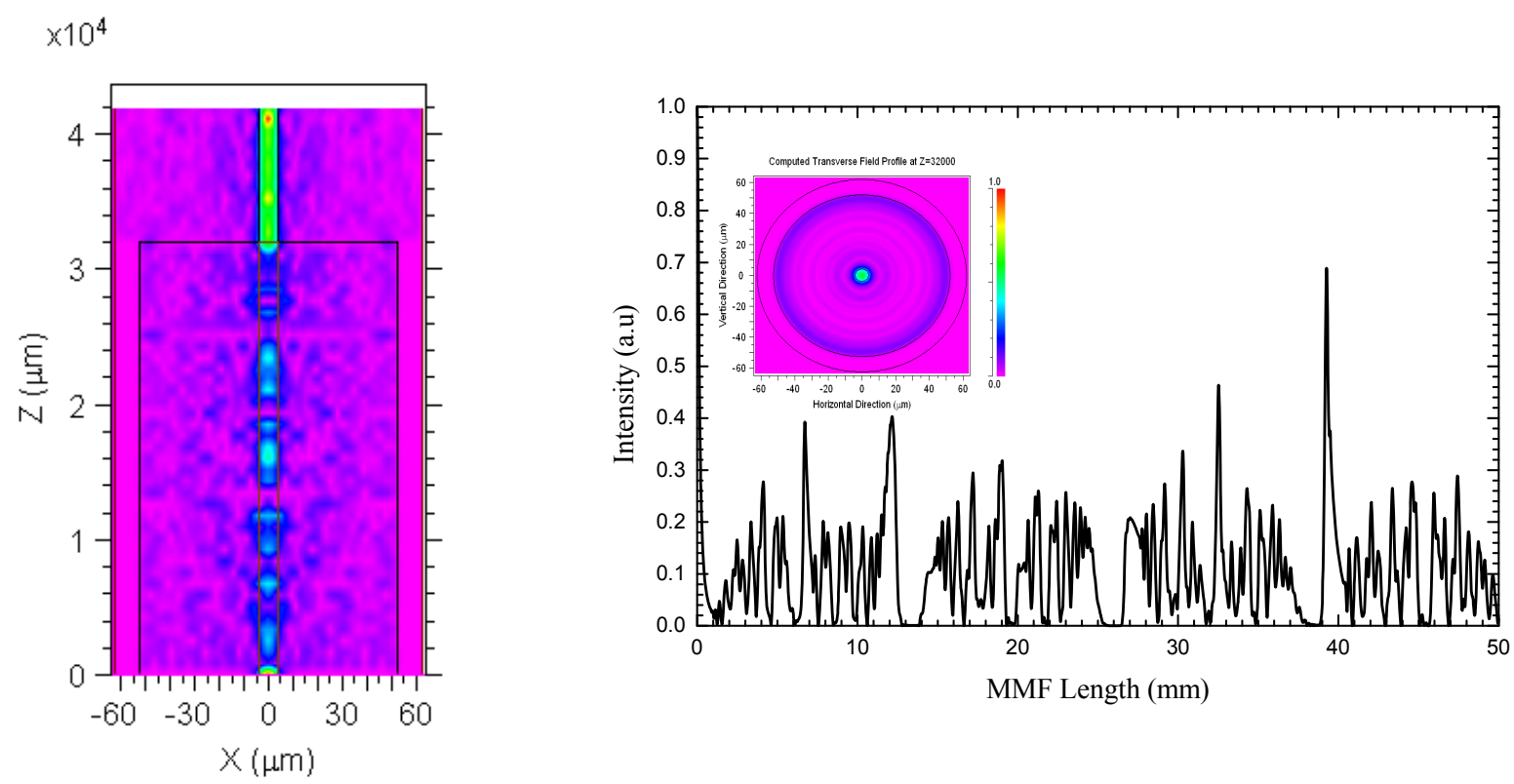

Figure 6. Left: Intensity distribution of electric field on the XZ plane, Right: coupling overlap integral between the calculated field at a given multimode fibre length and the input field for the simulation, a SMF-28 fundamental mode. Inset figure: the mode profile coupled into the output single-mode fibre.

Figure 7 presents the novel geometry of the Mach-Zehnder interferometer using multimode interference combined with a long period fibre grating. The MMI-LPG interferometer is composed by a multimode fibre with $32 \mathrm{~mm}$ of length; the distance between the MMF and the LPG is $300 \mathrm{~mm}$ that corresponds to the pattern fringe with the wavelength spacing of $2.7 \mathrm{~nm}$.

The piece of MMF couples the fundamental core mode and multiple forward-propagating cladding modes. A certain amount of the light propagating in the cladding mode is coupled back into the core by the LPG. Therefore, after the LPG two optical waves with a differential optical path delay propagate in the fibre core, resulting in an interference pattern that has the well-known channelled spectrum structure when broadband light is injected into the input fibre. 
An LPG with a period of $440 \mu \mathrm{m}$ and $21.6 \mathrm{~mm}$ length was arc-induced in Corning SMF-28 fibre. During the grating inscription, the fibre was kept under a tension of $5.1 \mathrm{~g}$, and was subjected to 20 arc discharges with $9 \mathrm{~mA}$ of current and 1 second duration. The obtained central wavelength for this condition was $1320 \mathrm{~nm}$.

The bend test is presented in Figure 7. A section of optical fibre $2 L=600 \mathrm{~mm}$ with the MMI-LPG in the middle was clamped between a translation stage and a fixed base. The sensor curvature is given by: $1 / R=2 d /\left(d^{2}+L^{2}\right)$, where $d$ is the bending displacement at the center of the MMI-LPG, $R$ is the curvature radius and $L$ is the half distance between the edges of the two clampers.

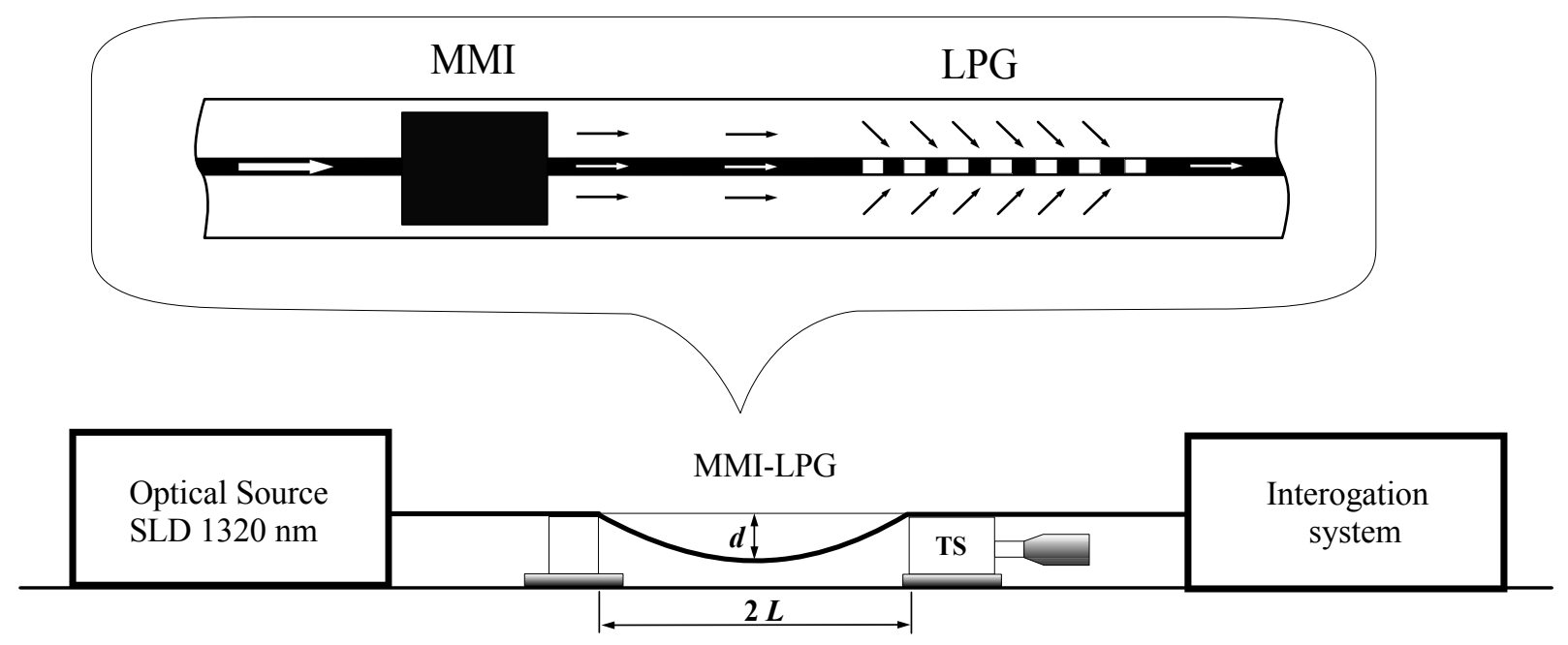

Figure 7. MMI-LPG geometry and the experimental setup to measure the bending.

The interrogation system for reading the MMI-LPG interferometer is based on white-light interferometer signal demodulation technique [18]. Figure 8 presents the measurement transmission power spectrum for the MMI-LPG interferometer. The signal includes the spectral response of the optical source (SLD $1300 \mathrm{~nm}$ ) with the pattern fringe of the MMI-LPG. The inset of Figure 8 shows the normalized spectral response of the MMI-LPG interferometer.

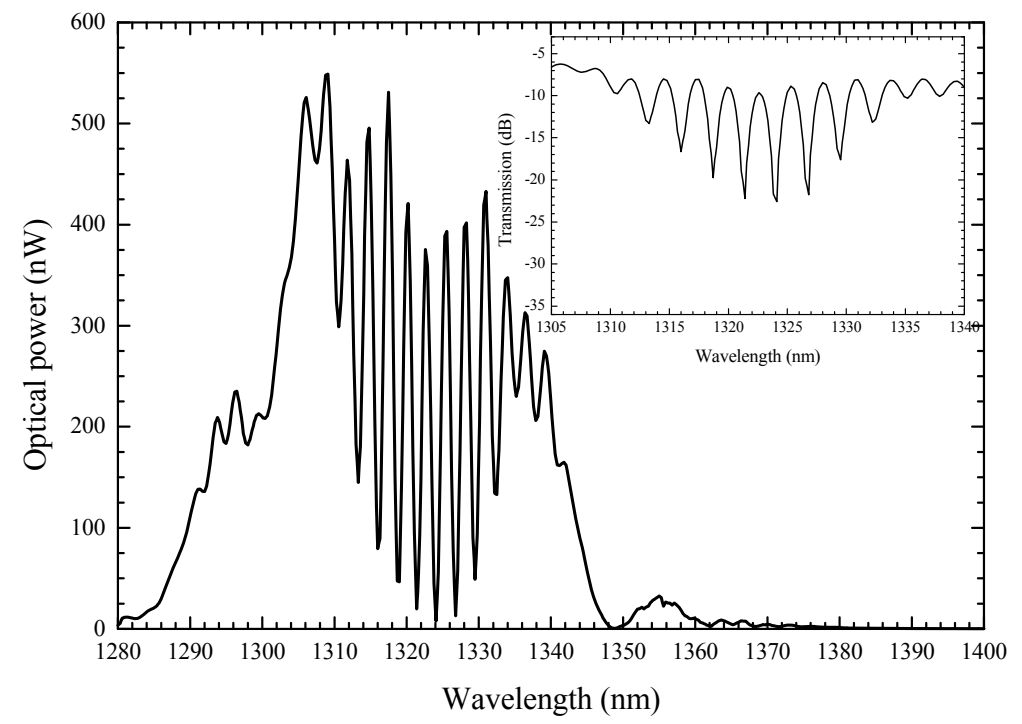

Figure 8. Spectral response of the optical source and the pattern fringe of the MMI-LPG. Inset figure: normalized transmission spectra of the MMI-LPG. 
Figure 9 shows the relationship between the phase shift of the MMI-LPG with the curvature radius. The range of the curvature radius is very large up to 18 metres. Inset Figure 9 presents the linear response of the curvature $(1 / R)$ and with the maximum error of $1 \%$. The phase shift is varied with the curvature radius due to MMI. When the MMI is bent, the optical path changes inside the MMI and so the phase of the interferometer. This can be understood by the high sensitivity of the MMI output on the asymmetry of effective index distribution on the radial coordinate caused by the bending.

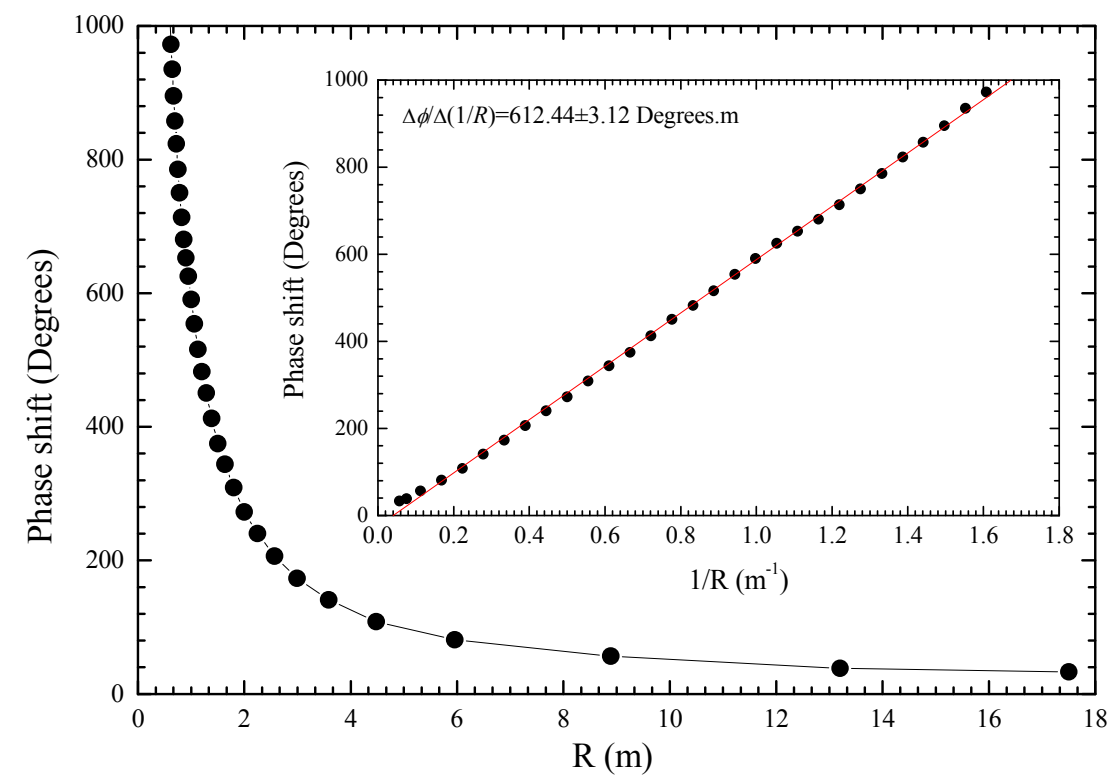

Figure 9. The relationship between the phase shift of the MMI-LPG with the curvature radius. Inset figure: function of the $1 / R$ corresponding to the curvature.

To summarize, in this section it was reported a Mach-Zehnder interferometer using a multimode interference combined with a long period fibre grating, with characteristics appropriate for operation over a large curvature range. The configuration is based on a piece of MMF combined with a LPG written by electric charge. An optimal $32 \mathrm{~mm}$ of MMF length was determined through simulation. A linear response of the curvature sensor of the MMI-LPG was obtained. It is envisaged that this sensing structure will find application in civil and mechanical engineering where large curvature can occur.

\subsection{Effect of Fiber Tapering in LPG-Based Mach-Zehnder Modal Interferometers for Refractive-Index Sensing}

In this section, an LPG-based Mach-Zehnder modal interferometer with a tapered fiber section between the two LPGs is studied as a sensing structure for measuring environmental refractive index.

The experimental set-up represented in Figure 10 was used to perform this study. Light from a super-luminescent diode (SLD), operating at $1320 \mathrm{~nm}$ with a FWHM spectral width of $\sim 35 \mathrm{~nm}$ (coherence length $\mathrm{L}_{\mathrm{c}} \approx 33 \mu \mathrm{m}$ ), was injected into the LPG-based Mach-Zehnder interferometer. This interferometer was assembled by placing in series two identical LPGs, with 3-dB transmission losses. 


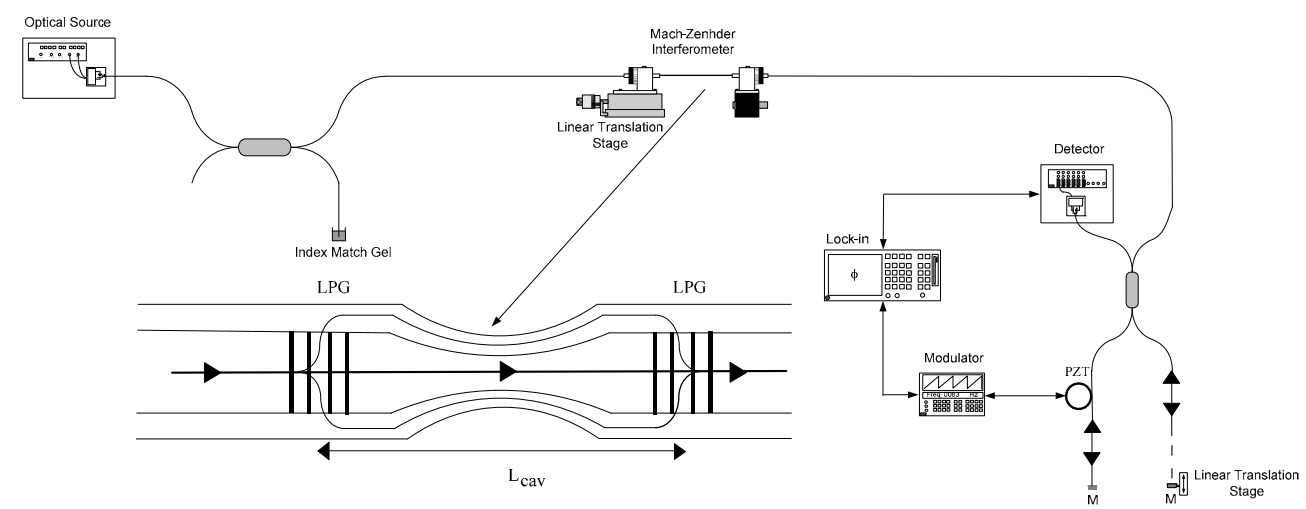

Figure 10: Experimental set-up showing the implemented interferometric interrogation scheme.

In this configuration, the first LPG couples a fraction of light to a specific cladding mode, whereas the remaining light keeps propagating in the fiber core and the second LPG couples the light back to the fiber core where it interferes with the light passing directly through the core. The core and cladding modes accumulate a differential optical path delay that is much larger than $\mathrm{L}_{\mathrm{c}}$. In particular, the cladding mode has a propagation constant that is dependent on the external refractive index, while the core mode provides a reference signal.

The LPGs were fabricated in-house using the electric-arc technique. The periods of the refractive index modulation were $476 \mu \mathrm{m}$ and $395 \mu \mathrm{m}$, values chosen to produce a resonance wavelength at approximately $1320 \mathrm{~nm}$, that match the SLD central wavelength, and corresponding to the 3rd and 5th cladding modes, respectively. The geometrical separation between the two LPGs without taper was $16 \mathrm{~cm}$. Various LPG-based Mach-Zehnder interferometers (using these two periods) were made with different separation between the LPGs without taper. The central part of each interferometer was tapered by heating and pulling the fiber using an electric arc and computer controlled translation stages. Several interferometers were obtained with different tapered lengths, all having the same initial separation between the two LPGs $(\sim 16 \mathrm{~cm})$.

To detect the phase changes in the LPG-based Mach-Zehnder interferometer, an optical fiber Michelson interferometer was built to implement coherence reading [18]. The Michelson interferometer was a conventional fiber Michelson interferometer with an open air path in one of its arms, which could be adjusted to match the optical path difference of the sensing interferometer.

Changes in the external refractive index could be applied by immersing the interferometer in samples of water mixed with different percentages of ethylene glycol. The refractive index of the different samples was calibrated using an Abbe refractometer with the sodium D line $(589 \mathrm{~nm})$, properly corrected for operation at $1320 \mathrm{~nm}$.

Any large shift in the LPG resonance would hinder the use of coherence addressing and pseudo-heterodyne processing technique, nevertheless, in a practical system this limitation can be easily overcome by assuring proper packaging of the LPG. Therefore, the LPGs were never bent during the experiments and the contact of the LPG with the liquid was prevented.

The phase response of the Mach-Zenhder interferometers was characterized submitting the sensing head to changes in the external refractive index in the range 1.33 to 1.42 . The sensitivity to external refractive index can be further improved by increasing the cavity length, and therefore, the interaction length of the LPG-based Mach-Zehnder interferometer. However, in many applications, such increase in the length of the sensing head can be a drawback. Interestingly, tailoring of the system sensitivity can also be achieved by introducing a tapered section in the interferometic cavity. The presence of the taper enlarges the span of the evanescent field of the selected cladding mode into the external medium. The increment is small but sufficient for significant enhancements of the sensitivity to external refractive index to be observed.

Figures 11 and 12 show how the sensitivity to external refractive index variations for each of the cladding modes investigated is affected by fiber tapering. 


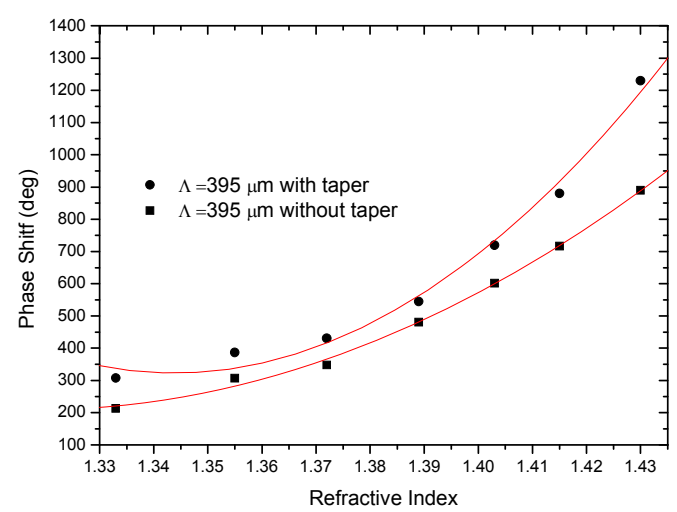

Figure 11: Sensing system phase changes due to refractive index variations for a Mach-Zehnder interferometer using a LPG with a period of $395 \mu \mathrm{m}$.

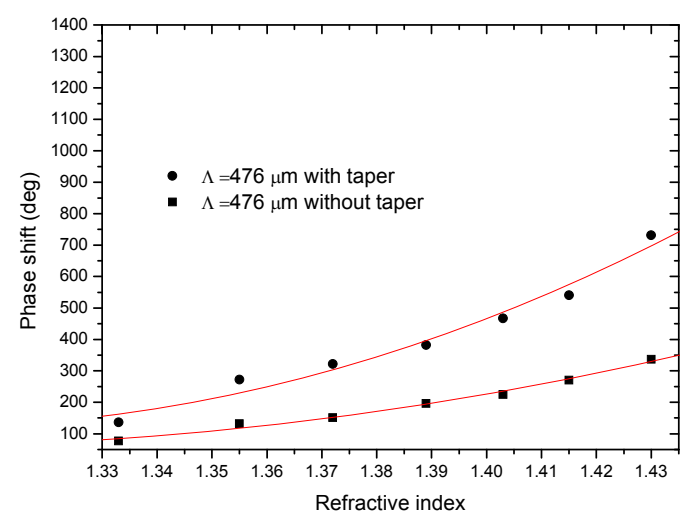

Figure 12: Sensing system phase changes due to refractive index variations for a Mach-Zehnder interferometer using a LPG with a period of $476 \mu \mathrm{m}$.

For both mode orders, the presence of a tapered section increases the sensitivity to external refractive index. In particular, in the refractive index region around 1.415, values of $19068 \mathrm{deg} / \mathrm{riu}$ and $\sim 10695 \mathrm{deg} /$ riu were obtained with and without taper, respectively, for the interferometer using a period of $395 \mu \mathrm{m}$, corresponding to an increase in sensitivity by a factor of $\sim 1.7$. For the interferometer using a period of $476 \mu \mathrm{m}$, in the same refractive index region, a sensitivity of $9920,5 \mathrm{deg} / \mathrm{riu}$ and $\sim 4164.1 \mathrm{deg} / \mathrm{riu}$ was obtained with and without the tapered section, respectively. In this case, the sensitivity increased by a factor of $\sim 2,5$. Comparison of the sensitivities of the different interferometers tested, indicate that the most sensitive device was the $5^{\text {th }}$ order mode $(\Lambda=395 \mu \mathrm{m})$ tapered interferometer. For the refractive index region around 1,415 the sensitivity of $5^{\text {th }}$ order tapered interferometer was 1,9 times higher than the $3^{\text {rd }}$ order tapered interferometer. However, the relative enhancement provided by the presence of a tapered section was more significant in the case of lower order modes.

The effect of taper length in the sensitivity to external refractive index using the LPG interferometers was also tested and the results are shown in Figure 13. For this experiment LPG-based Mach-Zehnder interferometers with a period of 476 $\mu \mathrm{m}$ were used, as the effect of tapering was more significant in these devices.

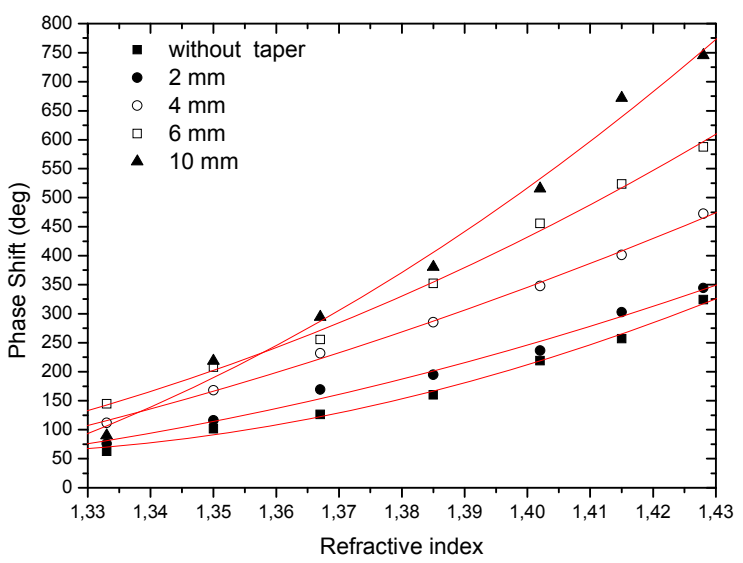

Figure 13: Sensing system phase changes due to refractive index variations for Mach-Zehnder interferometers with different tapered lengths using LPGs with a period of $476 \mu \mathrm{m}$.

Four different tapered sections with 2, 4,6 and $10 \mathrm{~mm}$ length were tested. The result shows that increasing the length of the tapered section increases the sensitivity to external refractive index changes. This result is clearly seen in Figure 14 where the phase difference accumulated when the interferometer is sequentially exposed to water (1.33) and ethylene glycol (1.42) is shown as a function of the tapered length. Sensitivity increases steadily with tapering length. 


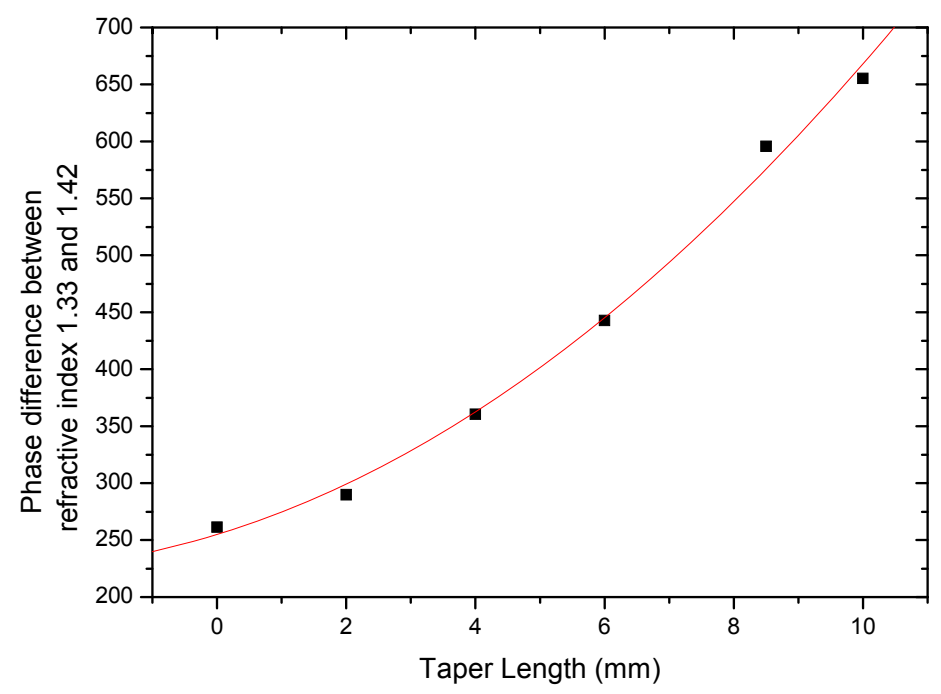

Figure 14: Sensitivity to external refractive index as a function of taper length.

The results obtained indicate the possibility of tuning the interferometer sensitivity by controlling mode order and tapering length, enabling the design of sensing heads that match the need of specific applications. This versatility opens the possibility of application of this structure for remote measurement of a large range of parameters, in particular biological and chemical entities.

\section{CONCLUSION}

Fibre optic interferometric structures have been largely explored in sensing due to the high sensitivities they permit in the measurement of a broad spectrum of parameters. Independently of the topological configurations they appear (Michelson, Mach-Zehnder, Fabry-Perot, Sagnac, etc.), different implementations have been considered aiming to optimize the device performance for a particular set of measurands. The group of modal interferometers, where the interferometric phase difference is built up considering the difference in the effective refractive index of different fibre modes, has been widely researched in the context of environmental sensing, curvature sensing and others. These structures are attractive due to several reasons, including small size and deployment flexibility, as well as the presence of a reduced thermal sensitivity in view of the usually small difference of the thermo-optic coefficients of the fibre modes under concern.

Globally, modal interferometry in optical fibers is a powerful concept when applied for sensing. Therefore, along the years, often triggered by technological developments, new realizations of it have been reported at an increasing rate. The consequence of this trend is a vast portfolio of fiber structures that can be applied to measure a large range of parameters at different environments. This brings a level of flexibility that, when combined with a remarkable performance, turns modal interferometry an appealing approach for fiber sensing. 


\section{REFERENCES}

[1] M. R. Layton, J. A. Bucaro, Optical fiber acoustic sensor utilizing mode-mode interference, Applied Optics, 18, 666-670. 1979.

[2] W. Eickhoff, Temperature sensing by mode-mode interference in birefringent optical fibers, Optics Letters, 6 , 204-206. 1981.

[3] A. Mehta, W. S. Mohammed, E. G. Johnson, Multimode interference-based fiber-optic displacement sensor, Photonics Technology Letters, 15, 1129-1131, 2003.

[4] Y. Jung, S. Kim, D. Lee, K. Oh, Compact three segmented multimode fibre modal interferometer for high sensitivity refractive-index measurement, Measurement Science and Technology, 17, 1129-1133, 2006.

[5] E. Li, X.Wang, C. Zhang, Fiber-optic temperature sensor based on interference of selective higher-order modes, Applied Physics Letters, 89, 091119, 2006.

[6] W. S. Mohammed, P. W. E. Smith, X. Gu, All-fiber multimode interference bandpass filter, Optics Letters, 31, 2547-2549, 2006.

[7] Q. Wang, G. Farrell, All-fiber multimode-interference based refractometer sensor: proposal and design, Optics Letters, 31, 317-319, 2006.

[8] O. Frazão, J. Viegas, P. Caldas, J. L. Santos, F. M. Araújo, L. A. Ferreira, F. Farahi, All-fiber Mach-Zehnder curvature sensor based on multimode interference combined with a long-period grating, Optics Letters, 32, 30743076, 2007.

[9] J. Zhang, Y. Zhang, W. Sun, L. Yuan, Multiplexing multimode fiber and Fizeau etalon: a simultaneous measurement scheme of temperature and strain, Measurement Science and Technology, 20, 065206, 2009.

[10] S. M. Tripathi, A. Kumar, R. K. Varshney, Y. B. P. Kumar, E. Marin, J.-P. Meunier, Strain and temperature sensing characteristics of single-mode-multimode-singlemode structures, Journal of Lightwave Technology, 27, 2348-2356, 2009.

[11] J. Zhang, S. Peng, A compact SMS refractometer based on HF corrosion scheme, presented at the Symposium on Photonics and Optoelectronic (SOPO), Chengdu, China, June 19-21 2010, pp. 1-4.

[12] Q. Wu, Y. Semenova, P. Wang, A. M. Hatta, G. Farrell, Experimental demonstration of a simple displacement sensor based on a bent single mode-multimode-single mode fiber structure, Measurement Science and Technology, 22, 025203, 2011.

[13] S. Silva, J. L. Santos, F. X. Malcata, J. Kobelke, K. Schuster, O. Frazão, Optical refractometer based on largecore air-clad photonic crystal fibers, Optics Letters, 36, 852-854, 2011.

[14] S. Silva, O. Frazão, J. Viegas, L. A. Ferreira, F. M. Araújo, F. X. Malcata, J. L. Santos, Temperature and strain independent curvature sensor based on a singlemode/multimode fiber optic structure, Measurement Science and Technology, 22, 085201, 2011.

[15] O. Frazão, P.Caldas, F. M. Araújo, L.A. Ferreira, J. L. Santos, Optical Flowmeter Using a Modal Interferometer Based on a Single Non-adiabatic Fibre Taper, Optics Letters, 32, 1974-1976, 2007.

[16] O. Frazão, J. Viegas, P. Caldas, J. L. Santos, F. M. Araújo, L.A. Ferreira, F. Farahi, All-fiber Mach-Zehnder Curvature Sensor Based on Multimode Interference Combined with a Long Period Grating, Optics Letters, 32, 3074-3076, 2007.

[17] P. Caldas, P. A. S. Jorge, F. Araújo, L. A Ferreira, G. Rego, J. L. Santos, Effect of Fiber Tapering in LPG-Based Mach-Zehnder Modal Interferometers for Refractive Index Sensing, $20^{\text {th }}$ International Conference on Optical Fiber Sensors, Proceedings SPIE 7503, 2009.

[18] D. Jackson, A. Kersey, M. Corke, J. Jones, Pseudoheterodyne detection scheme for optical interferometers, Electronic Letters, 18, 302-303, 1982. 